\title{
INFLAMMATORY MYOPATHY WITH ABUNDANT MACROPHAGES IN A JUVENILE SYSTEMIC LUPUS
} ERYTHEMATOSUS: A CASE REPORT

Sergio Luiz Oliveira Nunes (Faculdade de Medicina da Universidade de São Paulo (FMUSP), São Paulo, SP, Brasil), Marilia Ambiel Dagostin (Faculdade de Medicina da Universidade de São Paulo (FMUSP), São Paulo, SP, Brasil), Felipe Freire Silva (Faculdade de Medicina da Universidade de São Paulo (FMUSP), São Paulo, SP, Brasil), Douglas Amaral Moreira (Faculdade de Medicina da Universidade de São Paulo (FMUSP), São Paulo, SP, Brasil), Samuel Katsuyuki Shinjo (Faculdade de Medicina da Universidade de São Paulo (FMUSP), São Paulo, SP, Brasil), Rosa Maria Rodrigues Pereira (Faculdade de Medicina da Universidade de São Paulo (FMUSP), São Paulo, SP, Brasil)

\section{BACKGROUND}

Inflammatory myopathy with abundant macrophages (IMAM) is a rare entity and has been described in some systemic autoimmune diseases such as dermatomyositis and systemic lupus erythematous. To the best of our knowledge, the IMAM has not been reported before in juvenile systemic lupus erythematosus (jSLE).

\section{CASE REPORT}

A Caucasian 27-year-old female with jSLE since at the age of 17. She had previous clinical presentation of glomerulonephritis, seizures, autoimmune hemolytic anemia, urticarial vasculitis, hepatitis and serositis. Laboratory, she had ANA, anti-DNAds, anti-Sm, anti-ribosomal P, anti-Ro, and lupus anticoagulant. In early 2018 she had jSLE disease relapse (seizures, hepatitis and autoimmune hemolytic anemia), treated with methylprednisolone evolving with remission. In September 2018, she presented a new relapse (cutaneous lesions, autoimmune hemolytic anemia, increase of anti-DNAds titers, C3 and C4 consumption). Moreover, she had an intense myalgia and progressive difficulty to climb stairs, important weakness of pelvic and scapular girdle. Complementary exams showed: creatine phosphokinase serum level of 621U/L; electroneuromyography with predominantly proximal myopathy in the four limbs; magnetic resonance imaging with diffuse edema and enhancement of the muscular belly of the pelvic and thigh belts were observed, symmetrically, with peripheral and subfascial liquid laminae; muscle biopsy with an intense macrophagic inflammatory infiltrate in the perimisial and perifascial regions, with a mild CD8+ lymphocyte infiltrate and a moderate presence of necrotic fibers. Evaluation of infectious agents was negative for bacteria, fungi and mycobacteria. Induction therapy with methylprednisolone and human intravenous immunoglobulin pulse therapies were instituted, followed by maintenance with cyclosporine and prednisone. Clinical-laboratory improvement was obtained.

\section{CONCLUSION}

IMAM is a rare entity and this case shows that it should be considered in the differential diagnoses of jSLE patients with inflammatory myositis. 